\title{
Le territoire face à l'enjeu du développement durable
}

Le terme de territoire est devenu le maître mot des "nouvelles " politiques publiques. En France, depuis la décentralisation, on assiste à la prolifération des lois qui fondent leur modernité et leur efficacité sur le caractère territorial de leur application : Loi d'orientation sur l'administration territoriale (1992) qui crée les communautés de communes et de villes et le schéma départemental de coopération intercommunale ; loi d'orientation sur l'aménagement et le développement du territoire (1995 dite « loi Pasqua") qui instaure les "pays " et les directives territoriales d'aménagement ; loi du 12 juillet 1999 dite "Chevènement ", créant trois niveaux d'EPCl (établissements publics intercommunaux à fiscalité propre) : les communautés de communes, les communautés d'agglomération et les communautés urbaines; loi d'orientation sur l'aménagement et le développement durable du territoire (LOADDT 1999) qui confirme les " pays " et met au cœur de son caractère innovant le " projet de territoire ", en pariant sur le développement par le territoire des régions et des pays contractualisant avec l'État leur « projet de développement $»$; contrat territorial d'exploitation de la loi d'orientation agricole (2000). Actuellement, le projet de loi Raffarin met la " collectivité territoriale " au centre de l'amplification de la décentralisation... C'est que, sous couvert de territoire, la démocratie serait plus participative, plus adaptée au développement local, plus ascendante ou endogène. $C^{\prime}$ est aussi que le territoire serait l'instrument majeur du développement durable et en particulier " de la lutte contre la pauvreté " comme de la mise en œuvre de pratiques respectueuses des générations futures.

Mais l'usage du terme de territoire n'est-il pas abusif ? À quel titre le territoire est-il censé être garant d'un développement durable? Les territoires administratifs peuvent-ils être des " territoires de projet " ? Sont-ils des cadres adaptés pour un développement local, territorial et durable?

Soumis à l'analyse, les " territoires de projet " révèlent les risques de contradictions et d'incompatibilités entre les politiques territoriales. "À chaque projet son territoire ", dit-on : dès lors, les logiques spécifiques, et donc parcellaires, dominent; et il y a peu de chance pour qu'il en émerge la vision complexe et globale à la fois dans le temps et dans l'espace que suppose le développement durable. Les dimensions spatiales significatives pour les instances administratives, comme pour les porteurs de projet, sont à géométrie variable et le plus souvent en discor- dance. L'espace pour le développement d'un "pays " ne coïncide pas avec celui d'une prise en charge de la qualité de l'eau ; les "Schémas collectifs des espaces naturels et ruraux " découpés dans la perspective d'une planification régionale, ne correspondent pas davantage aux découpages socio-économiques ou environnementaux. Comment nouer entre elles les mesures diverses, les initiatives administratives européennes, nationales, régionales, les mesures agri-environnementales, la création des Parcs, la définition des Plans d'aménagement, des Chartes paysagères dans un projet de développement local ? Comment les mettre en connexion avec les initiatives locales spontanées, nombreuses, diverses, fragiles souvent, et qui sont plus ou moins combattues, acceptées, accompagnées ou soutenues par les institutions?

Deux conditions au moins, qui elles-même doivent être durables, sont requises pour que les territoires administratifs, qui sont des espaces de décision et d'intervention, facilitent l'émergence de territoires pouvant servir de base à un développement durable. La première est la création d'instances de dialogue et de concertation en constant renouvellement entre les acteurs du développement local ( $\mathrm{Cf}$. Hubert dans ce numéro). Cela suppose d'imaginer des procédures d'échanges, de négociations, et d'adapter celles qui existent. Pour éviter " l'étouffoir des mesures " (Cf. Abélès et al. dans ce numéro), rien ne peut être figé, ni les limites territoriales, ni les ressources locales à valoriser, ni les activités, ni les acteurs concernés.

La seconde condition, qui doit alimenter la première, est la production permanente de connaissances sur les mécanismes écologiques et sociaux qui sont mis en jeu - et en cause-dans le mouvement de développement local. Car le concept de territoire, central dans plusieurs disciplines, mérite d'être pris au sérieux et d'être instruit par le politique : les territoires ne sont pas seulement des espaces vécus et de sentiment d'appartenance, des aires de compétence, ce sont aussi des territoires physiques, cadastrés et appropriés, où s'affrontent les logiques d'acteurs et les enjeux. Utilisés comme base d'une activité cognitive partagée (Cf. d'Aquino dans ce numéro), outils de mesure des discordances et des conflits, ils pourraient être les terrains d'expérience inventant un développement durable.

Jean-Pierre Deffontaines, Nicole Mathieu 